\begin{tabular}{|c|c|}
\hline $\begin{array}{l}\text { Chemistry of } \\
\text { Metals and Allovs }\end{array}$ & 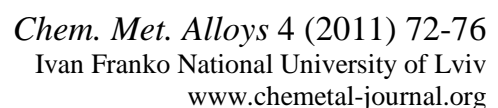 \\
\hline
\end{tabular}

\title{
Influence of thermal prehistory on the electrical properties of tungstate crystals
}

\author{
V.N. SHEVCHUK ${ }^{1}$, I.V. KAYUN ${ }^{1}$ \\ ${ }^{1}$ Faculty of Electronics, Ivan Franko National University of Lviv, Dragomanova St. 50, 79005 Lviv, Ukraine \\ * Corresponding author. Tel.: +380-32-394326; e-mail: shevchuk@electronics.wups.lviv.ua
}

Received July 7, 2010; accepted May 18, 2011; available on-line November 8, 2011

Temperature- and time-induced changes of electric and dielectric parameters of $A \mathrm{WO}_{4}(A=\mathrm{Cd}$, or $\mathrm{Pb})$ single crystals were studied experimentally. The dielectric properties measured along the [001] direction in $\mathrm{PbWO}_{4}$ single crystals (scheelite structure type) show a quasi-periodicity at about $20 \mathrm{~h}$ of free relaxation. A connection of this quasi-periodicity with structural and point defects dynamics, their interactions and the creation/rebuilding of complex defects (duration of the relaxation processes), is proposed. The main role in these phenomena is attributed to oxygen and lead vacancies. No thermodielectric effects were observed for $\mathrm{CdWO}_{4}$ (wolframite structure type) perpendicular to the cleavage face (010) up to $500 \mathrm{~K}$.

Tungstate crystal / Dielectric properties / Lattice defects / Relaxation

\section{Introduction}

Tungstate crystals $\mathrm{AWO}_{4}$ (where $A$ is $\mathrm{Pb}$ or $\mathrm{Cd}$ ) are intensively studied as efficient self-activated scintillation materials with short afterglow time [1]. The luminescence properties of $\mathrm{AWO}_{4}$ single crystals and their time characteristics depend on the crystal structural and point defects, the nature of which is complex and has not been established unambiguously. The electrical and dielectrical studies of these compounds are partial and insufficient, in spite of the fact that such data is highly informative as to the defects and their changes during the heat treatment.

Our previous works [2-4] on $\mathrm{PbWO}_{4}$ crystals regarding conductivity currents, thermally stimulated polarization, and depolarization currents within the temperature range $290-550 \mathrm{~K}$, have revealed a series of local energy levels and relaxation processes of different nature. The studies [2,3] of the permittivity $\varepsilon$ as a function of the temperature $T$ within the 290-550 K range showed a close connection between the temperature dependence and the depolarization processes in this compound.

In the present work electrically active defects in $A \mathrm{WO}_{4}$ crystals and their influence on characteristics of the crystals are investigated, taking into account the thermal prehistory of the crystals (maximal temperature of heating, polarization time, and time of free crystal relaxation). The dependence $\varepsilon(T)$ and thermally stimulated polarization / depolarization were considered for undoped $A \mathrm{WO}_{4}$ crystals with the purpose to elucidate regularities of defect formation, defect interactions, and ion-electron processes.

\section{Experimental}

Undoped $\mathrm{AWO}_{4}$ crystals obtained using the Czochralski technique were studied. The measurements were performed parallel to the growth axis [001] of the $\mathrm{PbWO}_{4}$ specimens (space group $\left.C_{4 h}^{6}-I 4_{1} / a\right)$, and perpendicular to the cleavage plane (010) of the $\mathrm{CdWO}_{4}$ crystal (space group $C_{2 h}^{4}-$ $P 2 / c)$. Fig. 1 shows details and crystallographic features of the experimental setup for the $A \mathrm{WO}_{4}$ crystals. Some crystallographic elements (basal, cleavage planes, labeled by the corresponding $h \mathrm{kl}$ indexes, intersections of the planes, structural elements, coordination polyhedra) of the investigated tungstate structures are also shown. Specimens of the dimensions $10 \times 10 \times 0.4 \mathrm{~mm}^{3}$ were prepared. Aquadag or Ag-paste was used as contact. A quartz temperature-controlled measuring cell was used in the investigations (with an accuracy of the temperature control better than $\pm 1 \mathrm{~K}$ ). As in our previous paper [2], the values of $\varepsilon$ were calculated assuming that the edge effects are small and using the well-known relation for a flat capacitor

$\varepsilon=C d / \varepsilon_{0} S$,

where $C$ is the measured capacitance of the sample; $d$ and $S$ are its thickness and area, respectively; and $\varepsilon_{0}$ is the permittivity of free space. The capacitance of the sample was recorded using a CLR E7-13 measuring unit (operation frequency $1 \mathrm{kHz}$ ). The root-meansquare of the voltage drop on a measured object did not exceed $0.2 \mathrm{~V}$. To obtain correct results, the capacitance of the connection system was taken into account. The temperature was measured with a 
standard chromel-alumel thermocouple. All the other experimental details were the same as those described earlier [2-4].

The measurements were performed in linear heating or cooling regimes at a rate of $0.1 \mathrm{~K} / \mathrm{s}$ in the temperature range $290-550 \mathrm{~K}$. Relaxation of the samples to a quasi-equilibrium state at room temperature after high-temperature heating was controlled by measuring the capacitance of the sample at certain time intervals $t$. In order to study the effect of preliminary excitation by an external dc electric field $E$, we applied a dc voltage (up to $50 \mathrm{~V}$ ) to a crystal over a time $t_{P}=5-15 \mathrm{~min}$ at different fixed temperatures $T_{P}$. The same model of spaceheterogeneous charge as previously [2,3], assuming blocking electrodes, is proposed. The experimental data was processed using the standard Origin software package.

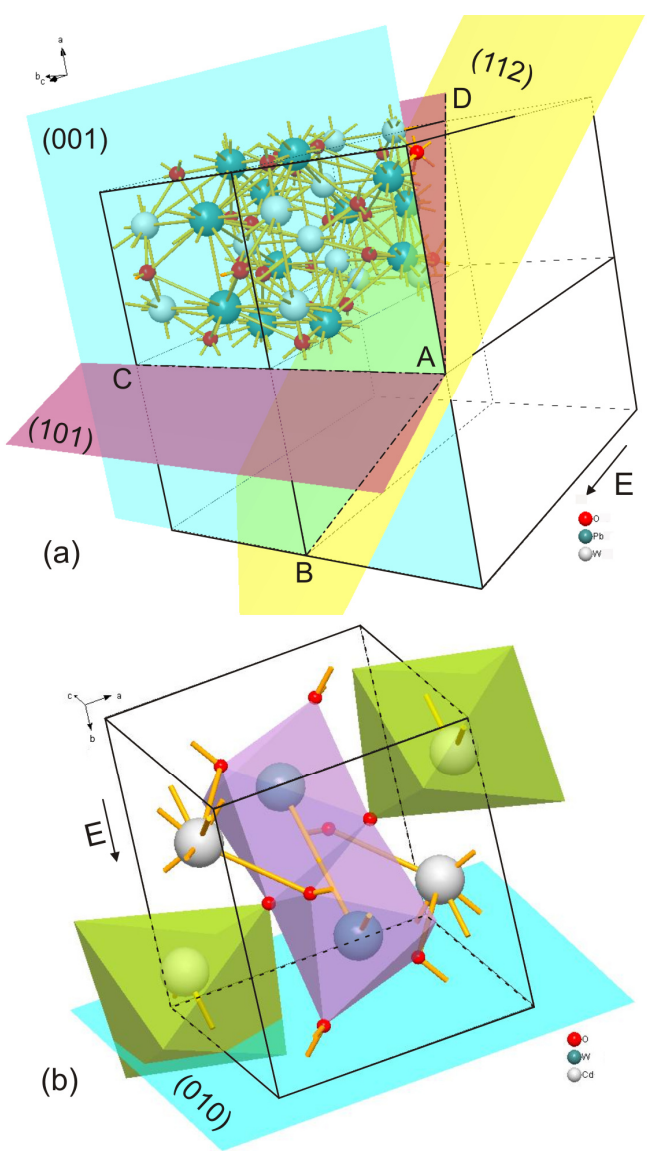

Fig. 1 Perspective view of the crystallographic unit cells of $\mathrm{PbWO}_{4}$ (a) and $\mathrm{CdWO}_{4}$ (b). Some elements of the structure are presented inside the unit cells. The $h k l$-indexes label the corresponding planes. The intersections of the planes are depicted by the $\mathrm{AB}, \mathrm{AC}$, and $\mathrm{AD}$ lines. The external electric dc field was applied parallel to the vector $\mathbf{E}$.
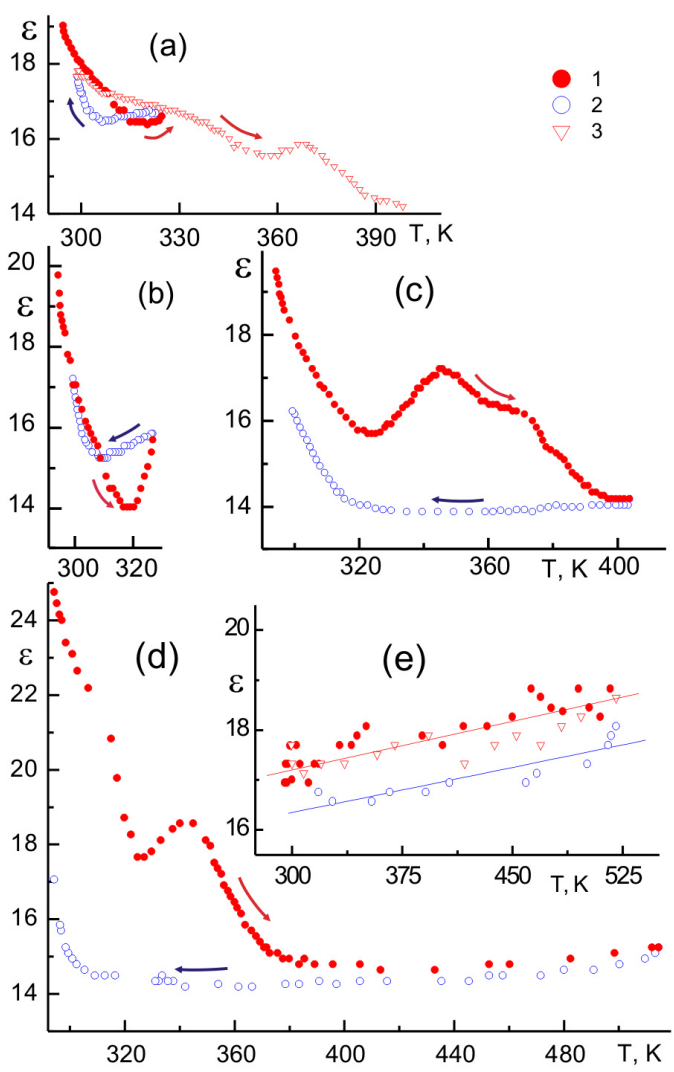

Fig. 2 Temperature dependencies of the permittivity of $\mathrm{PbWO}_{4}$ (a-d) and $\mathrm{CdWO}_{4}$ (e) crystals on heating (1) and cooling (2) for different maximum measurement temperatures $T_{h}$ in one heating-cooling cycle. Heating of the sample (3) immediately after the first cycle represented by curves 1 and 2. $T_{h}$ values: 325 (a), 335 (b), 405 (c), 515 (d), and $530 \mathrm{~K}$ (e). For $\mathrm{PbWO}_{4}$ (a-d) cycles of the measurements were performed after keeping the sample under normal conditions for longer than $24 \mathrm{~h}$.

\section{Results and discussion}

The dependence of the $\varepsilon(T)$ curve on the maximal heating temperature $T_{h}$ of the sample is presented for $\mathrm{PbWO}_{4}$ (Fig. 2(a-d)) and $\mathrm{CdWO}_{4}$ (Fig. 2e). The figure shows the curves of samples submitted to successive heating-cooling cycles. The temperature $T_{h}$ for the $\mathrm{PbWO}_{4}$ crystals was chosen in accordance with earlier investigations on thermally stimulated currents $[3,5,6]$. Using the thermal-depolarization method, under certain conditions we observed series of peaks of depolarization currents in the temperature ranges 290-350 K (range $A$ ), 350-380 K (B), and 400-600 K $(C)$. We assume that these peaks are related to dipole polarization (ranges $A, B$ ), or to space charges produced by equilibrium carriers in the sample (range $C$ ) $[3,5,6]$. 
In Fig. 2(a-d) we see that probable polarization effects strongly affect the $\varepsilon(T)$ curves in the ranges $A$ and $B$. In the range $400-520 \mathrm{~K}$, a conventional shape of the $\varepsilon(T)$ curve is observed (curve 1 in Fig. 2d). A nonlinear increase in $\varepsilon$ at high temperatures is caused by an exponential increase in conductivity with activation energies of $0.7-0.9 \mathrm{eV}[5,6]$ in the corresponding temperature intervals. In the range $290-400 \mathrm{~K}$, the $\varepsilon(T)$ curves measured in the heating mode exhibit closely spaced complicated maxima grouped in two rather narrow temperature regions, 290-330 and 330-400 K (curve 3 in Fig. 2a and curve 1 in Fig. 2(c,d)).

The $\varepsilon(T)$ curves measured on heating and cooling differ in shape. Thermal hysteresis appears for $T_{h} \geq 400 \mathrm{~K}$, where the values of $\varepsilon$ measured on heating are higher than those measured on cooling. In the cooling mode, no maxima are observed on the $\varepsilon(T)$ curve. In repeated experiments the $\varepsilon(T)$ curves measured on heating remain nonlinear, irrespective of $T_{h}$ or the duration of exposure of the sample to normal conditions prior to the measurements. At $T_{h} \geq 400 \mathrm{~K}$ (Fig. 2(a,b)), the values of $\varepsilon$ measured on cooling are greater than those measured on heating for the same temperatures.

At room temperature, the values of $\varepsilon$ usually lie in the range 25-30. These values agree with the data obtained for $\mathrm{PbWO}_{4}$ in $[7,8]$. For quasi-equilibrium values of $\varepsilon$ to be established after high-temperature heating, the sample must be kept under normal conditions for about $24 \mathrm{~h}$. The initial conductivity of the crystal is recovered after the same time [6].

At heating conditions of measuring, the function $\varepsilon(T)$ for $\mathrm{PbWO}_{4}$ is linear in the range $400-470 \mathrm{~K}$ and can be approximated by the relation

$\varepsilon=10.72+1.67 \times 10^{-3} \mathrm{~T}$.

On cooling within the range $470-320 \mathrm{~K}$, the function $\varepsilon(T)$ is also linear:

$\varepsilon=10.71+9.07 \times 10^{-4} \mathrm{~T}$.

For temperatures $T>470 \mathrm{~K}$, the experimental $\varepsilon(T)$ curves deviate from a linear dependence. This deviation is typical of most materials and possibly connected with electrical conductivity processes [5].

In the case of the $\mathrm{CdWO}_{4}$ crystal, the $\varepsilon(T)$ dependence is linear in the range 290-550 K (Fig. 2e), showing a slight linear increase according to the relationship

$\varepsilon=11.20+6.50 \times 10^{-3} T$.

At room temperature $\varepsilon=17$ for $\mathrm{CdWO}_{4}$. These values agree with the data reported in [9] $(\varepsilon=14)$. Practically no hysteresis was observed between the heating and cooling $\varepsilon(T)$ curves for $\mathrm{CdWO}_{4}$. Previous polarization of the crystal did not produce changes in the $\varepsilon(T)$ dependence.

Fig. 3 shows the influence of the external electric field $E$, temperature $T_{P}$, and thermal treatment on the $\varepsilon(T)$ curves of the $\mathrm{PbWO}_{4}$ crystal. Curves 1 and 2 show the results of the investigation of the effect of an external dc electric field on the $\varepsilon(T)$ dependence. For comparison, Fig. 3 also shows data for a non-polarized sample (curves 3,4). As the field was switched off at $290 \mathrm{~K}$, fast relaxation processes corresponding to $\varepsilon$ decreasing with time were observed. The $\varepsilon(T)$ function was measured after the end of the fast relaxation. The experiments show that, in the case of polarized samples, several maxima are observed in the $\varepsilon(T)$ curve in the range 290-430 K. The maxima near $320 \mathrm{~K}$ form a dominant, rather narrow peak. During the first measurement in the heating mode, this peak was found to be a few times higher than the corresponding peak in the case of a non-polarized sample. The peak intensities decrease after several heating-cooling cycles and free relaxation of the sample under normal conditions over 4-5 days. A sample polarized at $T_{P}=300 \mathrm{~K}$ and not heated retains high values of $\varepsilon=150-200$ at room temperature for a long time.

For non-polarized samples at $T \geq 420 \mathrm{~K}, \varepsilon$ slowly increases with increasing $T$. Polarization of the sample leads to a faster increase in $\varepsilon$ in the range $400-550 \mathrm{~K}$. After one or two cycles of heating to $T>400 \mathrm{~K}$ and subsequent cooling to room temperature, a nonpolarized sample returns by a two-stage way [2] to a quasi-equilibrium state with the initial value of $\varepsilon$.

Changes in the $\varepsilon(T)$ spectrum caused by irreversible transformations in the $\mathrm{PbWO}_{4}$ crystal during high-temperature heating are shown in Fig. 3b. Curve 1 was taken for the original sample with the initial concentration ratio of electrically active defects, for which models are considered in [2]. Heating to a

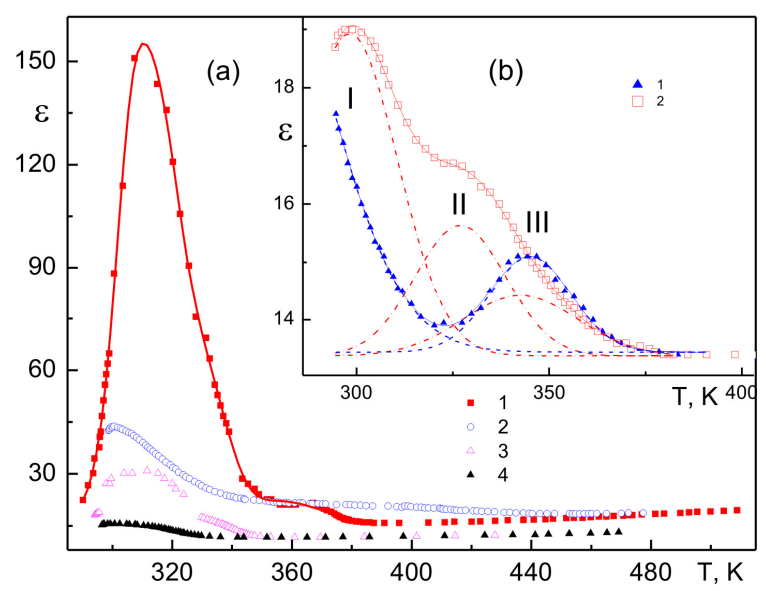

Fig. 3 Temperature dependencies of the permittivity (a) of an initially polarized $\mathrm{PbWO}_{4}$ crystal $\left(T_{p}=300 \mathrm{~K}, t_{p}=10 \mathrm{~min}\right) \quad(1)$, subsequent heating $\left(T_{p}=500 \mathrm{~K}, t_{p}=5 \mathrm{~min}\right)(2)$, and measurements on a non-polarized sample during two successive heating cycles $(3,4)$. The inset (b) presents the experimental curves of $\varepsilon(T)$ measured during the first heating (1), and after several heating-cooling cycles and relaxation at normal conditions (2). The dashed and dash-dotted lines show the elementary components I-III of curves 1 and 2, respectively, solid lines are the sum of the deconvolution components. 


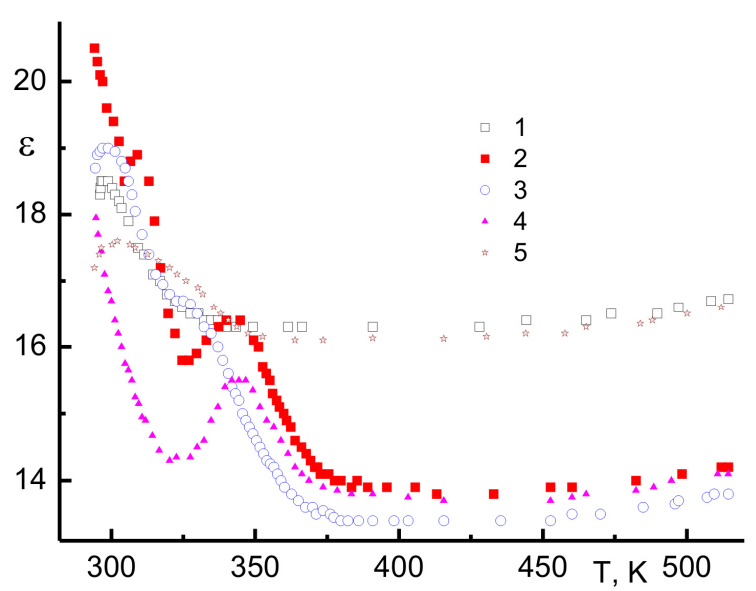

Fig. 4 Experimental function $\varepsilon(T)$ for $\mathrm{PbWO}_{4}$ crystals with different thermal prehistory. Curve (1) corresponds to $\varepsilon(T)$ obtained during the first heating; second heating after $24 \mathrm{~h}(2)$; third heating after $48 \mathrm{~h}(3)$; fourth heating after $72 \mathrm{~h}$ (4); fifth heating after $96 \mathrm{~h}(5)$.

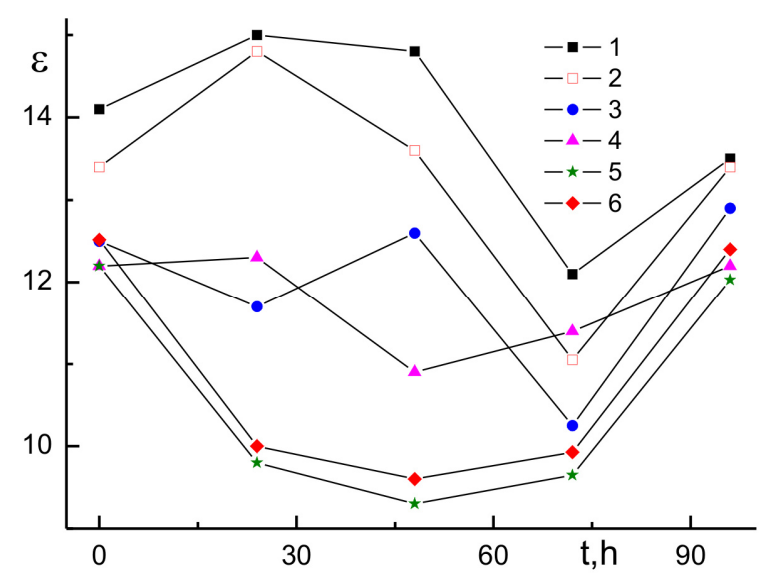

Fig. 5 Time evolution of $\varepsilon$ measured at selected (respectively to Fig. 4) temperatures: 302 (1), 310 (2), 320 (3), 345 (4), 400 (5), $500 \mathrm{~K}$ (6).

temperature $T>500 \mathrm{~K}$ changes this ratio by reducing the number of possible associate defects and the charge redistribution between different sorts of interacting capture centre. Curve 2 in Fig. 3 b shows the $\varepsilon(T)$ function for a sample subjected to such heating. In the subsequent cycles of heating the intensity components decrease (see Fig. 3b, curve 1). The component II is in this case not observed, and the third component shows almost no change. Only the components I and III were obtained from curve 2 by deconvolution. The decay of curve 2 is due to long relaxation processes (retention interval of $24 \mathrm{~h}$ under normal conditions).

The time evolution of the $\varepsilon(T)$ function and the values of $\varepsilon$ for $\mathrm{PbWO}_{4}$ at different temperatures are presented in Fig. 4 and Fig. 5, respectively. Curves 1-5 (Fig. 4) were taken under the same conditions at regular intervals ( 24 hours) of free sample relaxation at room temperature. The experiments testify about non-equilibrium of the sample and show that the ratio of the concentrations of certain types of electrically active defect, which appear due to interactions and quasi-chemical transformations of complex dipole defects under normal conditions, are changed. Under these conditions a difference in temperature is observed for the $\varepsilon(T)$ curves. The evolution of the samples as a function of time (Fig. 5) shows some quasi-periodicity of $\varepsilon$ values. This is illustrated by $\varepsilon(t)$ curves for selected $T$ below $400 \mathrm{~K}$.

The obtained experimental data testifies about significant differences between the electrical characteristics of the $A \mathrm{WO}_{4}$ crystals and their behaviour with respect to changes of temperature, thermal prehistory, and other experimental conditions. These differences are principally connected with structural features of the investigated crystals (see Fig. 1). $\mathrm{PbWO}_{4}$ crystals having the well-known cleavage plane (001), also cleave easily along the (101) and (112) planes under crack propagation [10]. The $\mathrm{CdWO}_{4}$ crystal has a perfect cleavage plane (010) [11]. The presence of the indicated planes determines / limits the ways of displacement of vacancy defects and capture of the latter by edge dislocations or other structural linear defects. In tungstate crystals grown by the Czochralski method a large number of dislocations are present. In $A \mathrm{WO}_{4}$ crystals the dislocation density is about $10^{3}-10^{6} \mathrm{~cm}^{-2}[12,13]$.

Edge dislocations are, as known from [14], charged. Migration of the dislocations at elevated temperatures or in an applied dc field (as shown in Fig. 1) is not excluded.

Interaction the dislocations with vacancies forms an irregular charge distribution in the crystal, local deformation instability, and leads to changes in the temperature and time dependencies of $\varepsilon$ and other characteristics of the sample. Under the influence of such processes the characteristic relaxation times of the sample will also change and the establishing of thermodynamic equilibrium can be of quasi-periodical character. For repeated heating-cooling cycles the processes will evolve towards the formation of micropores, until cracks appear in the crystal. Cracks were observed in our $\mathrm{PbWO}_{4}$ crystals after multiple thermal cycles. For the $\mathrm{CdWO}_{4}$ crystal, relaxation processes were not observed under the experimental conditions used here.

An additional mechanism that may change the $\varepsilon(T)$ function is the modification of the dipole moment of defect complexes in the process of thermal evolution [2]. Here the determining factor for changes in the sample is non-stoichiometry, whose role in cracking tungstate crystals was studied in [15].

Short- and long-term relaxation processes that can be controlled by measurement of electrical characteristics were observed in the $A \mathrm{WO}_{4}$ crystals. 


\section{Conclusions}

The scheelite structure type of $\mathrm{PbWO}_{4}$ is "more advantageous" to the lability of electrically active defects, in comparison with the wolframite structure type of $\mathrm{CdWO}_{4}$. In the $\mathrm{PbWO}_{4}$ crystal quasi-periodical changes of $\varepsilon$ and the thermo-dielectrical effect were experimentally detected at temperatures of measurement up to $400 \mathrm{~K}$ for the sample pre-excited by an electric field at elevated temperatures.

\section{References}

[1] M. Nikl, V.V. Laguta, A. Vedda, Phys. Status Solidi B 245(9) (2008) 1701-1722.

[2] V.N. Shevchuk, I.V. Kayun, Phys. Solid State 47(4) (2005) 632-637.

[3] V.N. Shevchuk, I.V. Kayun, Radiat. Meas. 42(4-5) (2007) 847-850.

[4] V.N. Shevchuk, I.V. Kayun, Acta Phys. Pol., A 117(1) (2010) 150-154.

[5] V.N. Shevchuk, I.V. Kayun, Phys. Solid State 45(10) (2003) 1898-1905.

[6] V.N. Shevchuk, I.V. Kayun, Funct. Mater. 10(2) (2003) 229-234.
[7] H. Huang, W. Li, X. Feng, P. Wang, Phys. Status Solidi A 187(2) (2001) 563-567.

[8] H. Huang, X. Feng, T.B. Tang, M. Dong, Z.-G. Ye, Phys. Status Solidi A 196(2) (2003) R7-R9.

[9] M.V. Mokhosoev, Zh.G. Bazarova, Complex Oxides of Molybdenum and Tungsten with Group I-IV Elements, Nauka, Moscow, 1990 (in Russian).

[10] I.P. Babiychuk, V.N. Baumer, V.G. Bondar, B.V. Grinyov, E.F. Dolgenkova, V.I. Krivoshein, E.N. Pirogov, V.D. Ryzhikov, Funct. Mater. 10(2) (2003) 224-228.

[11] L.N. Dem'yanets, V.V. Ilyukhin, A.V. Chichagov, N.V. Belov, Inorg. Mater. 3(12) (1967) 1938-1949.

[12] S. Ganesamoorthy, I. Bhaumik, A.K. Karnal, V.K. Wadhawan, J. Cryst. Growth 264 (2004) 320-326.

[13] B.P. Nasarenko, V.N. Baumer, E.F. Dolzhenkova, M.B. Kosmyna, Inorg. Mater. 41(10) (2005) 1114-1117.

[14] N.A. Tyapunina, E.P. Belozerov, Usp. Fiz. Nauk 156(4) (1988) 683-717.

[15] Sangeeta, S.C. Sabharwal, J. Cryst. Growth 310 (2008) 2899-2905.

Proceeding of the XVI International Seminar on Physics and Chemistry of Solids,

Lviv, June 6-9, 2010. 